\title{
H I shells in the outer Milky Way ${ }^{\star} \star \star$
}

\author{
S. Ehlerová and J. Palouš
}

\begin{abstract}
Astronomical Institute, Academy of Sciences of the Czech Republic, Boční II 1401, 14131 Prague 4, Czech Republic e-mail: sona@ig.cas.cz
\end{abstract}

Received 24 September 2003 / Accepted 7 March 2005

\begin{abstract}
We present results of a method for an automatic search for H I shells in 3D data cubes and apply it to the Leiden-Dwingeloo H I survey of the northern Milky Way. In the 2nd Galactic quadrant, where identifications of structures are not substantially influenced by overlapping, we find nearly 300 structures. The Galactic distribution of shells has an exponential profile in the radial direction with a scale length of $\sigma_{\mathrm{gsh}}=3 \mathrm{kpc}$. In the $z$ direction, one half of the shells are found at distances smaller than $500 \mathrm{pc}$. We also calculate the energies necessary to create the shells: there are several structures with energies greater than $10 E_{\mathrm{SN}}$ but only one with an energy exceeding $100 E_{\mathrm{SN}}$. Their size distribution, corrected for distance effects, is approximated by a power-law with an index $\alpha=2.1$. Our identifications provide a lower limit to the filling factor of shells in the outer Milky Way: $f_{2 \mathrm{D}}=0.4$ and $f_{3 \mathrm{D}}=0.05$.
\end{abstract}

Key words. ISM: bubbles - ISM: structure - Galaxy: structure - methods: data analysis

\section{Introduction}

The interstellar medium (ISM) in galaxies is far from homogeneous: it is turbulent and composed of several coexisting phases. This creates a complex picture, so far not fully understood. Important constituents of this picture are structures known as shells, supershells and holes (usually called this in association with observations in $\mathrm{HI}$ ) or bubbles and superbubbles (in connection with $H_{\alpha}$ or other wavelengths). These structures are known to exist in the Milky Way Galaxy and many external galaxies (for a summary of observations of shells see Walter \& Brinks 1999). Most probably they are created by energy release from massive stars (winds and supernova explosions), however, alternative explanation, gamma-ray bursts (GRBs; Efremov et al. 1998; Loeb \& Perma 1998) or high velocity cloud (HVC) infalls (Tenorio-Tagle \& Bodenheimer 1988), have been invoked in some cases. The majority of the observed shells are due to star formation. Structures connected with the infall of HVC are exceptional (Ehlerová \& Palouš 1996). The origin of shells in connection with GRBs has not been proven (Efremov et al. 1999). A connection between massive stars and H I shells can be tested either by observation of individual objects or by a comparison of statistical properties such as galactic or size distributions. In this paper we choose the second method.

\footnotetext{
* Appendix is only available in electronic form at http://www.edpsciences.org

$\star \star$ Table 1 is only available in electronic form at the CDS via anonymous ftp to cdsarc.u-strasbg.fr $(130.79 .128 .5)$ or via http://cdsweb.u-strasbg.fr/cgi-bin/qcat?J/A+A/437/101
}

There are many papers on shells in the Milky Way but the majority of them are concerned with individual objects. The most notable exceptions are two papers by Heiles $(1979,1984)$ which contain lists of shells in the northern Galaxy, the paper by $\mathrm{Hu}$ (1981) on northern high latitude shells, and more recently the paper by McClure-Griffiths et al. (2002) on shells in the southern Galaxy. Unfortunately, none of these lists are complete, but are the best data available for the Milky Way. In this paper we try to create a more complete list of shells in our Galaxy. We use the Leiden-Dwingeloo H I survey and create an automatic algorithm to find shells. Then we compare identified structures with shells in external galaxies and discuss their Galactic and size distributions.

The structure of the paper is as follows: we start with a short description of the dataset used for identification (Sect. 2), continue with a description of the identification method (Sect. 3) and other searching methods (Sect. 4). Then we analyse results for the 2nd galactic quadrant of the Milky Way (Sects. 5 and 6) and summarise our findings (Sect. 7). In the Appendix we try to cross-identify the shells found with those published previously.

\section{Data}

We use the Leiden-Dwingeloo H I survey (Hartmann \& Burton 1997; LDS). The survey was made in 1989-1993 with a $25 \mathrm{~m}$ radio telescope in Dwingeloo (The Netherlands). It covers $79 \%$ of the sky with a spatial resolution of $0.5^{\circ}$, a velocity resolution of $1.03 \mathrm{~km} \mathrm{~s}^{-1}$ and an rms noise of $0.07 \mathrm{~K}$.

We chose this survey because of high sky coverage and uniformity of data. LDS has not been searched for shells up to now. Contrary to many H I surveys used to find shells, 
like the Canadian Galactic Plane Survey (CGPS), LDS covers the whole sky, not only a strip around the Galactic equator. Therefore we are able to study shells with large angular sizes $\left(\Delta b>10^{\circ}\right)$ and also the distribution of shells at high latitudes.

The automatic method of shell detection that we describe in this paper is quite general and can be applied to any data cube. It is obvious that by using a data cube with a higher angular resolution (such as CGPS) we would discover more smaller structures than by using LDS. However, as it will be shown later (e.g. Fig. 4), angular dimensions of many discovered shells exceed the region covered by CGPS. Consequently, they would remain undiscovered if we did not use an allsky survey. Moreover, a structure with a dimension of $500 \mathrm{pc}$ (which is quite typical) has an angular dimension larger than $5^{\circ}$ at a heliocentric distance less than $5.7 \mathrm{kpc}$. Therefore, as we are primarily interested in shells with dimensions of several $100 \mathrm{pc}$, the LDS (or any other high-coverage, low-resolution survey) is appropriate. If we were interested in smaller shells, like windblown bubbles, a high-resolution survey (such as CGPS) would be better.

Our position inside the Milky Way makes the identification more difficult compared to external galaxies because of substantial overlapping of material along the line-of-sight, because of a linear resolution that changes with heliocentric distance and because of problems with distance determination.

\section{Searching algorithm}

A prototypical ideal $\mathrm{HI}$ shell has several signatures which distinguish it from the background: it is a region of decreased H I emission surrounded by a dense thin wall, it is expanding and its shape is spherical. The reality is more complex: the ISM is stratified and turbulent; $\mathrm{HI}$ shells evolving in this medium are elongated and irregular, their expansion slows down during the evolution; and there are usually intervening material, gradients induced by galactic rotation, and the finite resolution of the telescope, all of which render the identification of shells more difficult. Taking the difficulties mentioned above into consideration we conclude that the most persistent quality of an $\mathrm{HI}$ shell is the existence of a region with decreased $\mathrm{HI}$ emission, e.g. an $\mathrm{HI}$ hole. Our automatic algorithm is based on this conclusion and it searches for holes.

The identification procedure has three steps: 1) a search in separate velocity channels; 2) a merging of structures in adjacent velocity channels to form an H I shell candidate; and 3) a check using the central pixel spectrum.

\subsection{The search for holes in separate velocity channels}

We search for regions of local low brightness temperature completely encircled by higher temperature surroundings. Not all pixels inside the encircled region must belong to the hole, but all participating pixels must form a continuous structure. For each local minimum we search for the maximum encircling temperature, i.e. the one defining the maximum region that fulfills the described condition. Figure 1 shows different types of prototypical structures; in the upper row there are structures

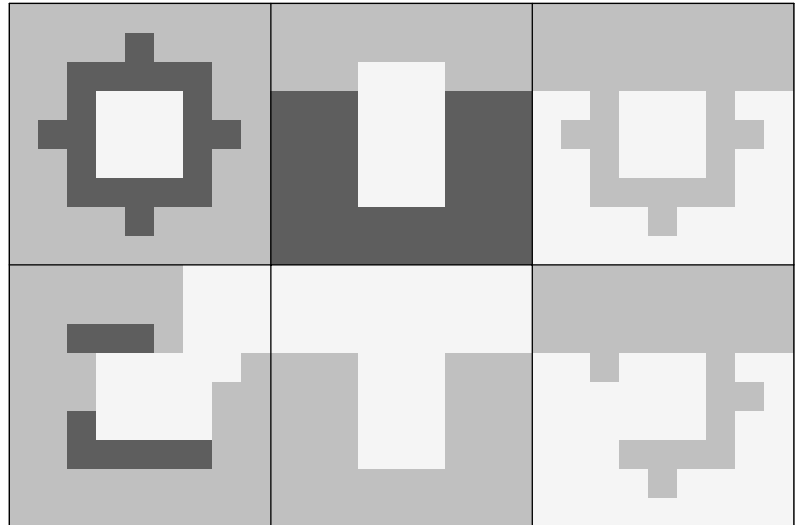

Fig. 1. Types of H I shells. The first row shows examples of structures, that can be identified, the second row shows structures connected to the surroundings or with incomplete walls, which are undetectable by the code.

detectable with our algorithm, in the lower row there are structures that are not detectable. The first case is a local depression surrounded by a wall, which has a higher density than the medium around it (Fig. 1, upper left). In some cases the wall is not observed and the structure is just a local depression in the H I gas. The second case (Fig. 1, upper center) is a local depression in the medium with a steep density gradient. This case is known as a galactic H I worm and the structure is frequently perpendicular to the galactic disc because of the largescale $z$-stratification of the H I. The last type is an arc blown out into a low density environment (Fig. 1, upper right).

All three described cases are detectable with our search algorithm. The difficulty begins when the local depression is directly connected to other low density regions (Fig. 1, lower left). The search algorithm is unable to close the structure. Thus, it either joins it to a larger neighboring hole or does not identify it at all. Worms, which are open at one or both ends connecting regions where the HI emission is absent (Fig. 1, lower center) are undetected as well. The identification also fails for fragmented arcs in a medium with low H I emission (Fig. 1, lower right). Examples from the LDS of the cases described are given in Fig. 2.

The only constraints put on the hole are its dimensions: the hole must be greater than a minimum dimension and smaller than a maximum dimension. We use the range of dimensions $\left(1.5^{\circ}, 45^{\circ}\right)$. There are no constraints on the shape of $\mathrm{HI}$ holes.

In this paper we search in $l b$ channel maps. $P V$ diagrams can be used as well, however, many shells are open in velocity diagrams (i.e. only one hemisphere is visible) and this kind of structure is not well suited to our type of search. A full 3D search is time-consuming and also suffers from the mentioned disadvantages of $P V$ diagrams.

\subsection{The formation of an $\mathrm{HI}$ shell candidate}

After finding holes, i.e. continuous regions around local minima in individual velocity channels, substantially overlapping holes in consecutive radial velocity channels are automatically joined together to form a 3D structure. If the structure joins four 

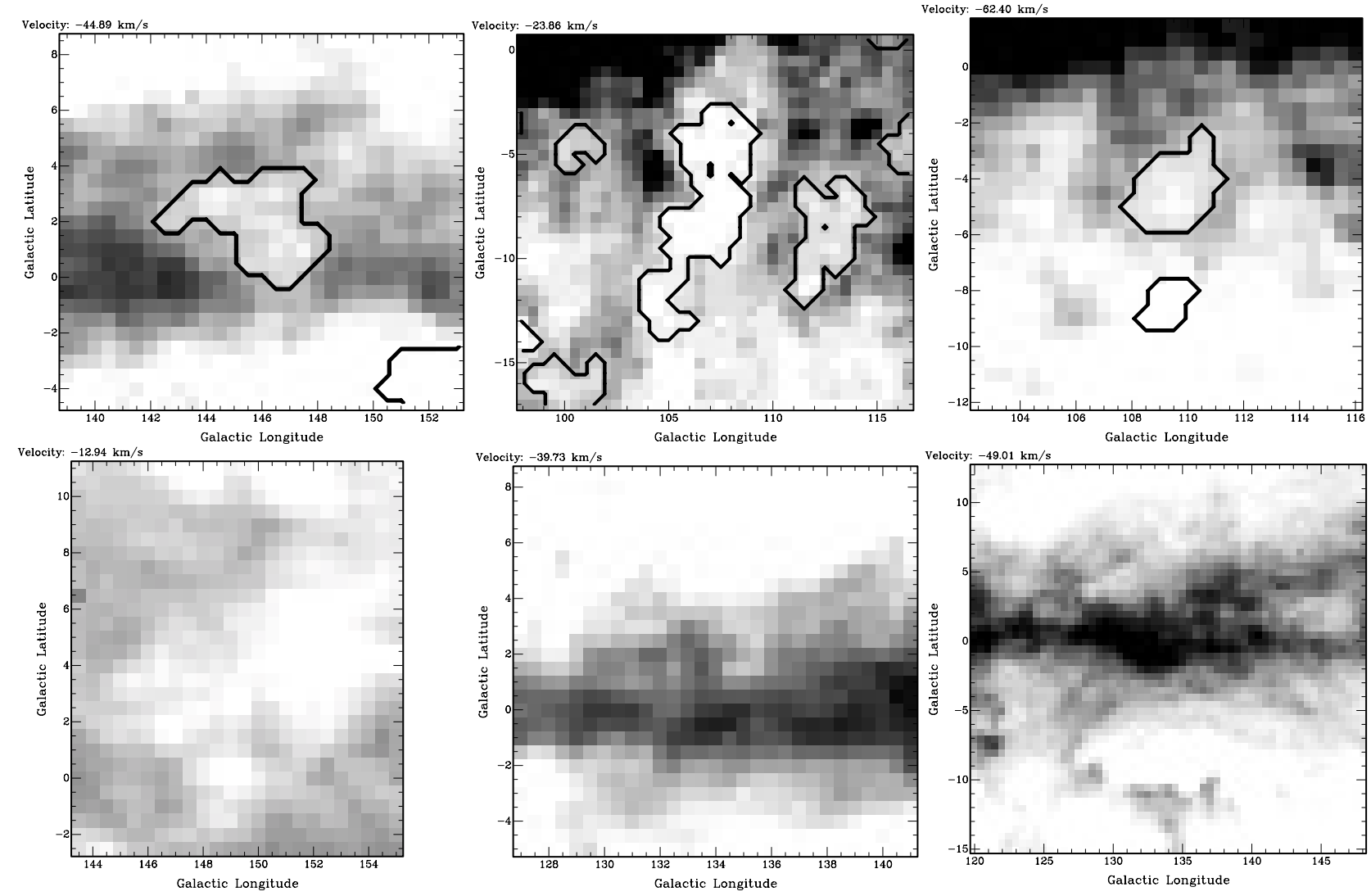

Fig. 2. H I shells in the LDS corresponding to different types as shown in Fig. 1.

or more velocity channels (i.e. if its velocity extent is larger than $4 \mathrm{~km} \mathrm{~s}^{-1}$ ), it is called an $\mathrm{HI}$ shell candidate. In the majority of cases the shell extends also to a few adjacent velocity channels, in which the angular size is less than $1.5^{\circ}$, and therefore it is not identified by our algorithm in step 1 (but it can be identified in step 3, see below). If only four or five consecutive velocity channels are involved, the velocity difference between parts of the HI shell candidate is less than the typical velocity dispersion in the ISM: $\sigma_{\text {ISM }}=5-7 \mathrm{~km} \mathrm{~s}^{-1}$. In that case, we probably see a static structure, which will be dissolved after the dissolution time $t_{\mathrm{diss}} \sim r_{\mathrm{sh}} / \sigma_{\mathrm{ISM}}$, where $r_{\mathrm{sh}}$ is the size of the shell.

\subsection{Verification using the central pixel spectrum}

As a third step in the identification scheme the spectrum through the center of the structure is automatically checked. The coordinates of the center $\left(l_{\mathrm{c}}, b_{\mathrm{c}}, v_{\mathrm{LSR}}\right)$ are mean values of all pixels forming the $\mathrm{HI}$ shell candidate. $\mathrm{A} \Delta T_{\mathrm{B}}=T_{\text {hole }}-T_{\mathrm{bg}}$ spectrum through the center of an $\mathrm{HI}$ shell candidate is analyzed for depressions and peaks. The background brightness temperature $T_{\mathrm{bg}}$ is defined in each velocity channel as an average emission from a strip around the structure with $b=b_{\mathrm{c}}$. This definition proved to be more satisfactory than other tested methods, e.g., the average emission from the surroundings of the structure.

Structures that do not contain a clear depression in the $\Delta T_{\mathrm{B}}$ spectrum or for which the depression is not located in the velocity interval where the hole is visible in $l b$ maps are excluded from further study. Figure 3 shows examples of spectra. Structures in the upper row are identified in velocity channels where the $\Delta T_{\mathrm{B}}$ spectrum has a minimum, therefore all are HI holes. The upper left gives the spectrum showing a minimum in those velocity channels where the HI shell candidate is identified (thick dashed line). The upper center shows a more complex spectrum with several smaller peaks in velocity channels corresponding to a temperature depression of the HI shell candidate. They probably come from small cloudlets engulfed by the HI hole. The upper right gives an example of a spectrum with a wall, which closes the HI hole at the position of the central pixel. On the other hand the three spectra in the lower row do not clearly demonstrate an H I structure. The spectrum in the velocity channels where the HI shell candidate resides is either too flat (left) or the identified structure is displaced from the minimum in $\Delta T_{\mathrm{B}}$ (center) or the structure is not connected to the minimum in $\Delta T_{\mathrm{B}}$ (right).

The interval $\Delta v$ between peaks surrounding the depression in $\Delta T_{\mathrm{B}}$ or between endpoints of upward slopes if peaks do not exist, equals twice the expansion velocity of the structure $v_{\text {exp }}=\frac{1}{2} \Delta v$. The $\Delta v$ interval is usually larger than the velocity interval where the hole is visible in $l b$ maps. This is because the algorithm searches for holes (i.e. minima in $\Delta T_{\mathrm{B}}$ ) and moreover for holes with dimensions larger than a given size. Therefore, velocity channels that contain low-contrast or small parts of the structure are not included in an $\mathrm{H}$ I shell candidate created in the first two steps of the identification. Only a minority of 


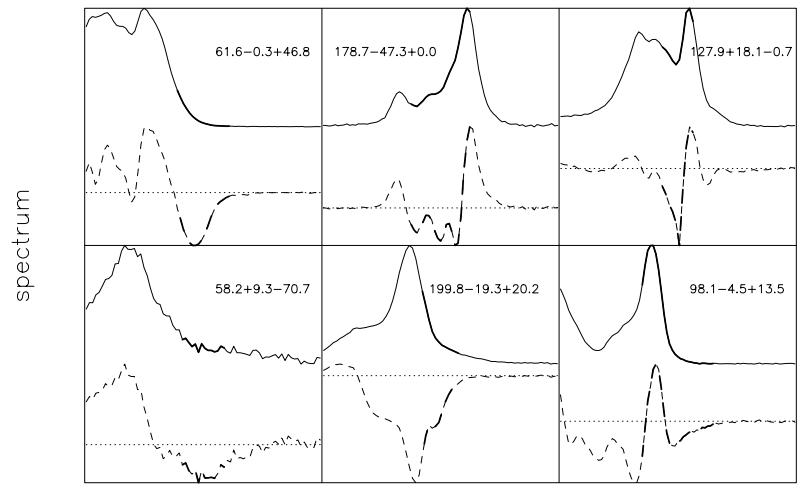

velocity

Fig. 3. Spectra through the central pixel of H I shell candidates. The upper row shows spectra where the presence of an H I hole is confirmed. The lower row shows spectra where the signature of an $\mathrm{H}$ I hole is not clear or not present. Solid lines are observed $T_{\mathrm{B}}$ spectra, dashed lines are $\Delta T_{\mathrm{B}}$ spectra, a dotted line shows the background level. Velocity channels in which the HI shell candidate (steps 1 and 2) are detected are plotted by the thick line. Numbers are coordinates of structures $(l b v)$.

structures has an interval $\Delta v$ from the spectrum analysis smaller than the visibility interval. This is usually because several substructures exist inside the hole, e.g. interacting shells, or preexisting H I clouds. In such a case it is difficult to decide which peak or upward slope corresponds to the rim of the structure.

\subsection{Tests on artificial data}

Tests with artificial data show that completeness in identification of high-contrast shells is good regardless of gradients in the background. Low-contrast shells in the rapidly changing background can be misidentified as being smaller, or are completely missed. Small shells (compared to the thickness of the disc) are more easily identified than large ones. Random brightness temperature fluctuations create structures with a small velocity extent, which are easily removed when checking the reality of the feature by analyzing a spectrum taken through the central pixel.

When extrapolating these results from artificial data to types of real shells we expect that the completeness of our identification for younger (i.e. not blown-out and not very distorted) shells is quite high, while older shells, which are blown-out and have fragmented walls, can be missed. We cannot detect shells that are formed by incomplete arcs: we detect only structures with a hole inside or completely encircled regions.

\section{Other automatic searches for $\mathrm{H}$ I shells}

Another approach in the search for H I shells uses models of expanding structures (usually hydrodynamic simulations) and then it searches in the observed data cubes for patterns similar to artificial data from models. This method was applied by Thilker et al. (1998) and Mashchenko et al. (1999) for H I shells in the galaxy NGC 2403, or by Mashchenko \& St-Louis (2002), who use a simple analytical model to search for H I counterparts of a few H II regions in CGPS. Calculated shapes of $\mathrm{HI}$ shells are usually quite smooth and regular, due to a smooth density distribution in the model. More complicated structures with irregular walls and noisy velocity patterns, which are results of the turbulent nature of the ISM and of preexisting H I structures, remain undiscovered by this method. Our approach does not use any precomputed shapes and it can discover rather complicated forms. The only limitation is the angular and velocity resolution and the sensitivity limit.

The second approach primarily uses dynamical characteristics of expanding shells (Daigle et al. 2003). It searches for a signature of an expansion in the velocity spectra, i.e. the existence of two peaks separated approximately by twice an assumed expansion velocity. Regions with these features are then tested if their appearance corresponds to an assumed shape of shells. This method was applied to a small field of CGPS data, where it found two expanding bubbles.

In our method the spectrum through the center of an H I shell candidate is tested after the region is chosen as a depression in $l b$-maps, which is one difference between the methods. The other difference is that we do not prefer any expansion velocity and we do not require the existence of peaks in a spectrum, which is a more general condition. We do not put any restrictions on the shape. The spectrum analysis serves as a way to distinguish between random configurations of pixels and physical structures.

The method as a whole is quite robust. It is not sensitive to the adopted model of a shell, as is the first approach described above, and the spectrum through the structure need not contain peaks, as is the prerequisite of the method used by Daigle et al. (2003). Our approach resembles the reversed method of de Heij et al. (2002) used for identification of high velocity clouds in LDS.

The third - but from the chronological point of view the first - method is a traditional identification by eye. With the growing amount of data and increasing resolution this method becomes more difficult and more time-consuming. Visual identification, compared to automatic methods, is not very effective for smaller and more regular or semi-regular shells. It is, however, so far unsurpassed when it comes to very irregular, patchy, non-expanding and open structures because of the ability of the human eye to combine disconnected features into a single shape.

\section{H I shells in the Leiden-Dwingeloo survey}

In the whole LDS we have identified more than 600 structures that passed the three steps in our identification scheme. Their positions on the sphere are shown in Fig. 4 and their list is given in Table 1, which is available at the CDS. The table contains the following information: Col. 1 - the shell number, Col. 2 - the shell name in the form GSlll $\pm b b \pm v v v$, Col. 3 - the galactic longitude of the center $l_{\mathrm{c}}$ in degrees, Col. 4 - the galactic latitude of the center $b_{\mathrm{c}}$ in degrees, Col. 5 - the radial velocity of the center relative to the local standard of rest $v_{\text {LSR }}$ in $\mathrm{km} \mathrm{s}^{-1}$, Col. 6 - the shell dimension in the galactic longitude direction $\Delta l$ in degrees, Col. 7 - the shell dimension in the galactic latitude direction $\Delta b$ in degrees, Col. 8 - the shell extent in radial velocity 


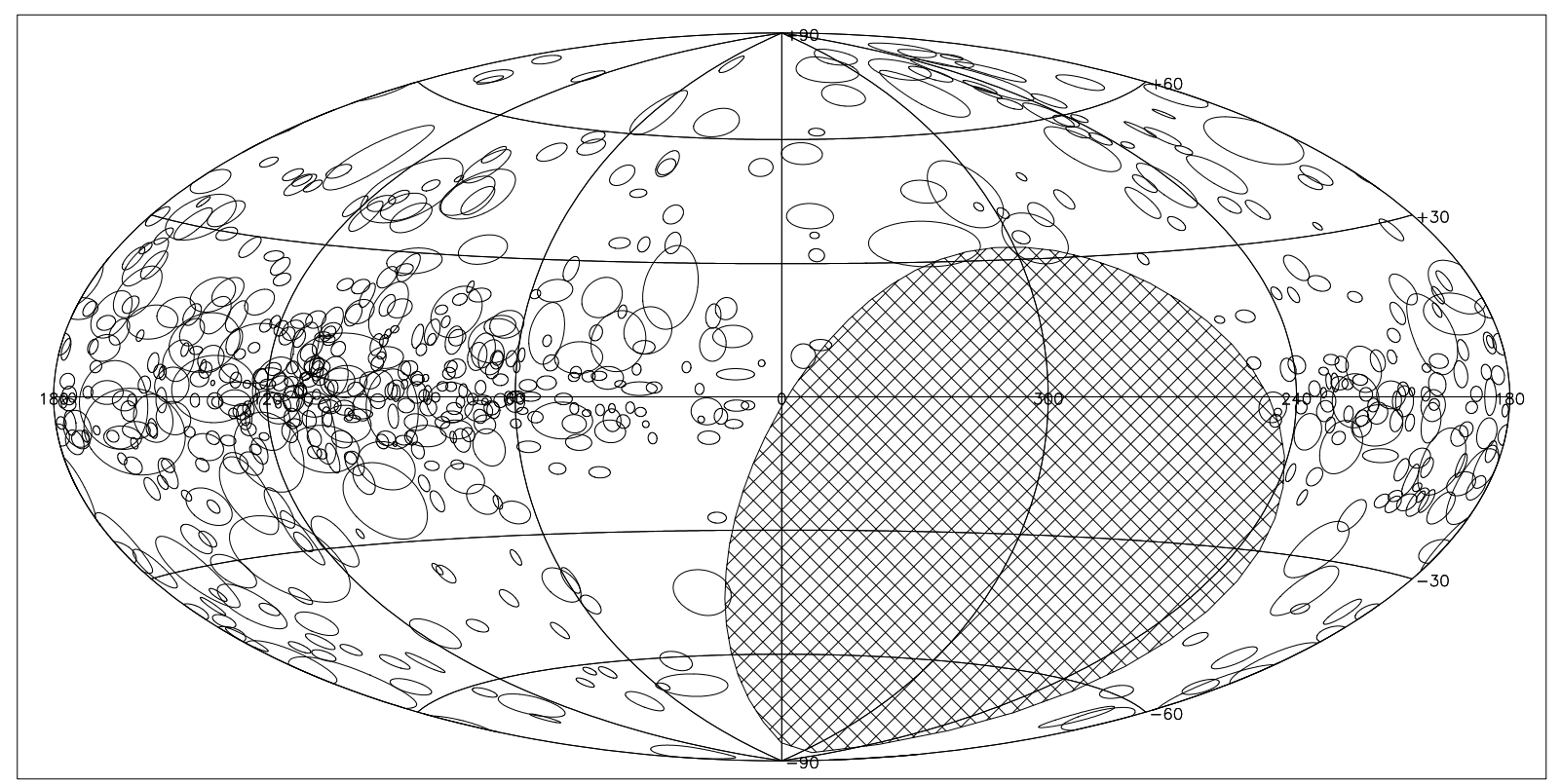

Fig. 4. Positions of identified HI shells in the LDS. The cross-hatched region around $l=300^{\circ}$ is a region not accessible to the Dwingeloo telescope.

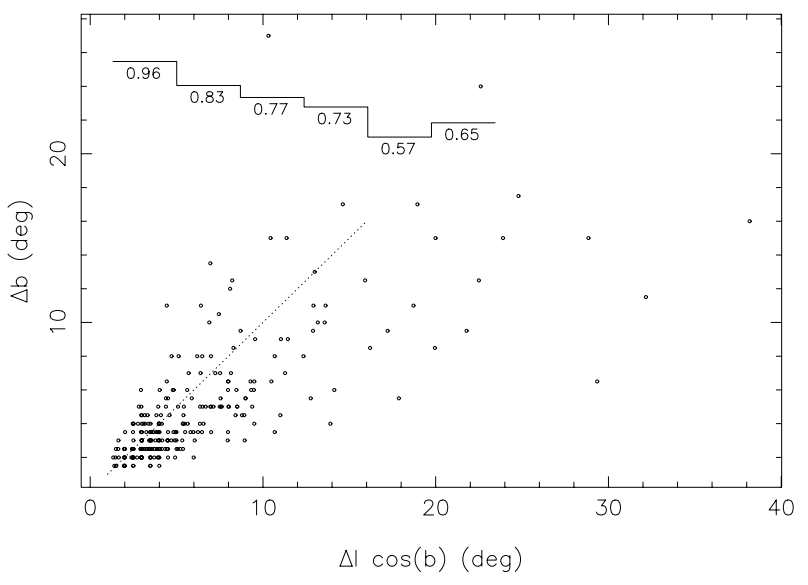

Fig. 5. Angular dimensions $\Delta b$ against $\Delta l \cos b$ of shells identified in the 2nd Galactic quadrant. The dotted line corresponds to $\Delta b=$ $\Delta l \cos b$. The upper solid line gives the average values of $\Delta b / \Delta l \cos b$ in $\Delta l \cos b$ bins.

$\Delta v$ in $\mathrm{km} \mathrm{s}^{-1}$, Col. 9 - a quality index, Col. 10 - a corresponding structure identification in other lists of H I shells. Notes (1) and (2) in Col. 10 are explained in the ReadMe file of the table. The quality index is based on the visual appearance of the structure and it is not part of the automatic search. A quality index of 1 denotes clearly visible and pronounced shells, while an index 4 refers to low-contrast and not well-pronounced shells. Indices 2 and 3 are intermediate steps.

There is substantial overlap of shells in the inner galaxy, which causes incompleteness of the identification. To avoid its influence on the results we decided to restrict further analysis to $276 \mathrm{HI}$ shells discovered in the 2nd galactic quadrant. Moreover, the 2nd quadrant is fully covered in LDS.

The majority of structures have $\Delta l \cos b>\Delta b$ (see Fig. 5), although there is a population of $b$-elongated structures (upward and to the left of the dotted line in Fig. 5). Some of them are worms. All large shells $\left(>15^{\circ}\right)$ are, with one exception, more elongated in the $l$ direction than in the $b$ direction. This can partly be a result of the incomplete identification: because of the blowout effect the large shell is open to high $z$, and only a part of the structure is identified. This leads to an artifically lower $\Delta b$.

The ratio $\Delta b / \Delta l \cos (b)$ is a good estimate of the shape of shells parallel or perpendicular to the Galactic equator. This ratio is not a reliable parameter for very irregular shells or shells elongated in directions other than the $l$ or $b$.

We calculated kinematic distances $d$ using the Wouterloot et al. (1990) rotation curve. Some shells have forbidden velocities or lie close to the anticenter direction. This reduces the number of shells in the 2nd quadrant with kinematic distances to 168 . The angular dimensions $\Delta l \cos b, \Delta b$ are transformed to linear sizes. The shell radius $r_{\mathrm{sh}}$ is given as

$r_{\mathrm{sh}}=\frac{1}{4} d[\tan (\Delta l \cos b)+\tan (\Delta b)]$.

The dimensions of structures as a function of heliocentric distance are shown in Fig. 6. The solid lines indicate the limits imposed on $r_{\mathrm{sh}}$ by restricting the range of angular sizes of the shells to the interval $(1.5,45)$ degrees. Let us assume that the size distribution of shells has a universal power-law form (Oey \& Clarke 1997):

$\mathrm{d} N\left(r_{\mathrm{sh}}\right) \propto{r_{\mathrm{sh}}}^{-\alpha} \mathrm{d} r_{\mathrm{sh}}$,

where $\mathrm{d} N$ is the number of shells with radius $r_{\mathrm{sh}} \in\left(r_{\mathrm{sh}}, r_{\mathrm{sh}}+\right.$ $\mathrm{d} r_{\text {sh }}$ ). For shells with the size distribution (2) we calculate the expected average size for a given heliocentric distance $d$ as

$\bar{r}_{\mathrm{sh}}=\frac{\int_{r_{\min }}^{r_{\max }} r^{-\alpha+1} \mathrm{~d} r}{\int_{r_{\min }}^{r_{\max }} r^{-\alpha} \mathrm{d} r}$. 


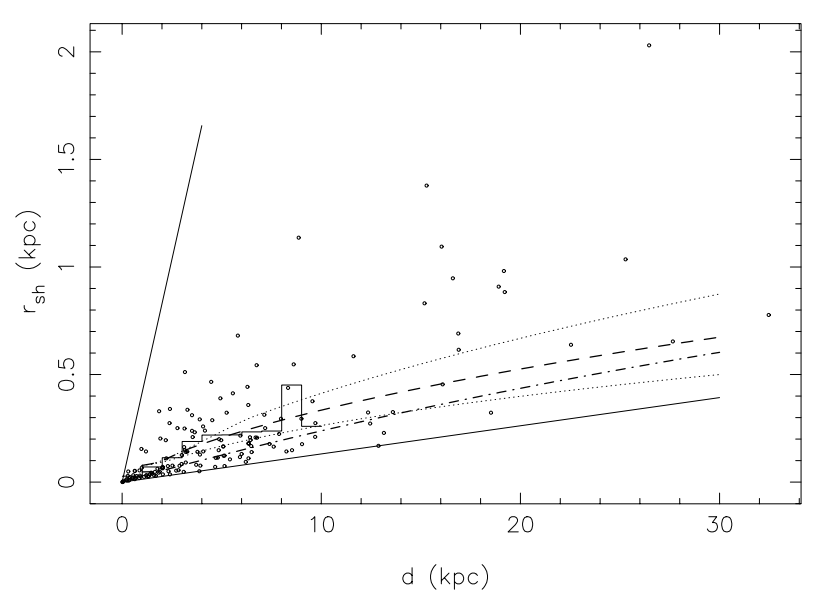

Fig. 6. Radius of the shells $r_{\mathrm{sh}}$ versus their kinematic distances. The two solid lines give the minimum and maximum $r_{\mathrm{sh}}$ corresponding to $1.5^{\circ} / 2$ and $45^{\circ} / 2$ at the distance $d$. Dashed, dotted and dash-dotted lines give the average $r_{\mathrm{sh}}$ calculated for different models of size distribution of shells given by Eq. (2). The histogram gives the average radius $r_{\mathrm{sh}}$ of the observed shells in separate distance bins.

Integration ranges $r_{\min }$ and $r_{\max }$ are given either by real physical limits (minimum and maximum possible radius of the shell) or by the resolution limits $\left(d \tan 1.5^{\circ} / 2, d \tan 45^{\circ} / 2\right)$ of the searching algorithm, whichever is appropriate (larger or smaller). The major part of the theoretical curves in Fig. 6 uses $r_{\min }=d \times \tan 1.5^{\circ} / 2$ and $r_{\max }=1.3 \mathrm{kpc}$ (if not said otherwise; for the choice of $1.3 \mathrm{kpc}$ see Oey \& Clarke 1997). The thick dashed line in Fig. 6 refers to the size distribution with $\alpha=2$; the thick dot-dashed line to $\alpha=3$. Thin dotted lines have $\alpha=2$ and $r_{\max }=2.6 \mathrm{kpc}$ (the upper line) or $r_{\max }=0.65 \mathrm{kpc}$ (the lower line). According to a comparison with the histogram in Fig. 6, showing the average observed shell radii in separate distance bins, it seems that the power-law with $\alpha=2$ and the maximum radius of the shell $r_{\mathrm{sh}}=1.3 \mathrm{kpc}$ best fits the observations, but the spread in $r_{\mathrm{sh}}$ is large.

The distribution of expansion velocities $v_{\exp }=\frac{1}{2} \Delta v$ is shown in Fig. 7. The largest observed expansion velocity is $25 \mathrm{~km} \mathrm{~s}^{-1}$; the median value lies between 5 and $10 \mathrm{~km} \mathrm{~s}^{-1}$. There are only a few structures with $v_{\exp }<5 \mathrm{~km} \mathrm{~s}^{-1}$, which is apparently because such non-expanding structures cannot survive for very long in a turbulent ISM (see Sect. 3.2).

\subsection{Radius vs. expansion velocity}

A relation between the shell radius $r_{\mathrm{sh}}$ and the expansion velocity $v_{\text {exp }}$ is shown in Fig. 8. We also plot lines of constant expansion time and luminosity over the ISM density $(L / n)$ according to an analytical solution of Weaver et al. (1977). As expected, there are no shells younger than $1 \mathrm{Myr}$ and there are only a few shells with ages greater than about 50 Myr. Young shells are small and not fully developed, while old shells are either completely destroyed by external forces (like spiral arms) or too fragmented for our method to identify. There is a lack of small shells with high expansion velocities, but this is not surprising. Shells smaller than about $50 \mathrm{pc}$ are either wind-blown bubbles or remnants after one supernova explosion. Such objects tend

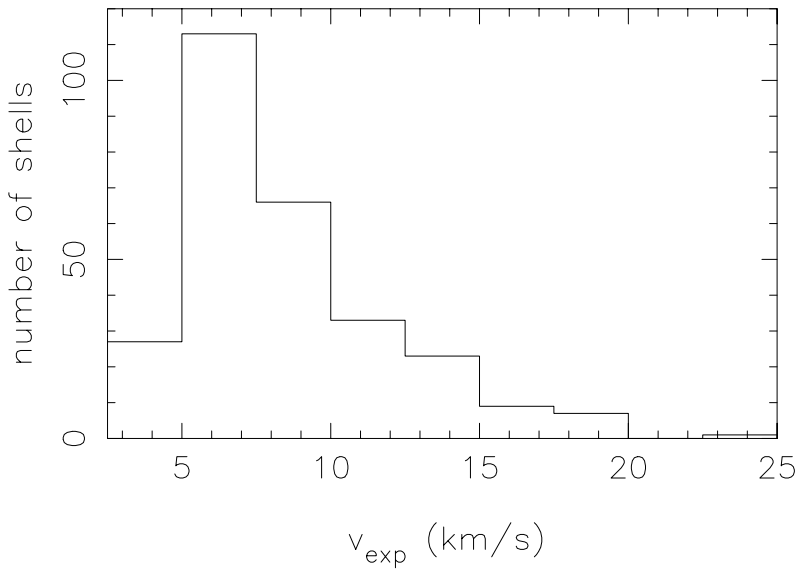

Fig. 7. Expansion velocities of identified H I shells in the 2nd Galactic quadrant.

to have expansion velocities around or lower than $10 \mathrm{~km} \mathrm{~s}^{-1}$. Dimensions of real "supershells", created by several OB stars in associations, start at radii around $100 \mathrm{pc}$. Some supershells have expansion velocities larger than $10 \mathrm{~km} \mathrm{~s}^{-1}$, though we do not see any clear dependence of radius on velocity. The argument that small shells are wind-blown bubbles and large shells are created by several SNs is further supported by their energies (Chevalier 1974). All small shells have energies lower than the typical energy of one supernova explosion $E_{\mathrm{SN}}=10^{51} \mathrm{erg}$.

It is interesting to compare the properties of shells in the Milky Way with those in external galaxies. Walter \& Brinks (1999; further WB99) made a comparison of four galaxies, M 31, M 33, IC 2574 and Holmberg II. They plot a graph of radius vs. expansion velocity (Fig. 20 in their paper) for these four external galaxies. They find that the two spiral galaxies (M 31 and M 33) have on average smaller shells and a larger range of expansion velocities compared to the dwarfs IC 2574 and Ho II. Neither dwarf contains relatively small shells (radius around 100-300 pc) with high expansion velocities (around $20 \mathrm{~km} \mathrm{~s}^{-1}$ ), while there is a significant population of such shells in M 31 and M 33. The Milky Way behaves like a spiral galaxy in this aspect; there are many expanding shells with a 100-300 pc size. Contrary to M 31 and M 33, in the Milky Way there are more shells with radii greater than $800 \mathrm{pc}$, while there is only one in M 31 and a few in M 33. This can be partly due to a different inclination angle of galaxies, because different viewing angles may emphasize different parts of shells. This effect is only important for non-spherical, therefore predominantly large shells.

Differences in the $r_{\text {sh }}$ vs. $v_{\text {exp }}$ graph can be explained by different conditions between spiral and dwarf galaxies and by different star formation histories. It is possible to calculate the age of a shell from its size and expansion velocity, e.g. using the solution of Weaver et al. (1977), see Fig. 8. According to WB99, spiral galaxies M 31 and M 33 have many young shells (younger than $10 \mathrm{Myr}$ ), but nearly no old ones (older than 30 Myr), while dwarf galaxies IC 2574 and especially Ho II have few young shells, but many old ones. The mean ages of shells in these galaxies are 6 Myr (M 31), 7.2 Myr (M 33), 14 Myr (IC 2574) and 37 Myr (Ho II); all quoted values were 


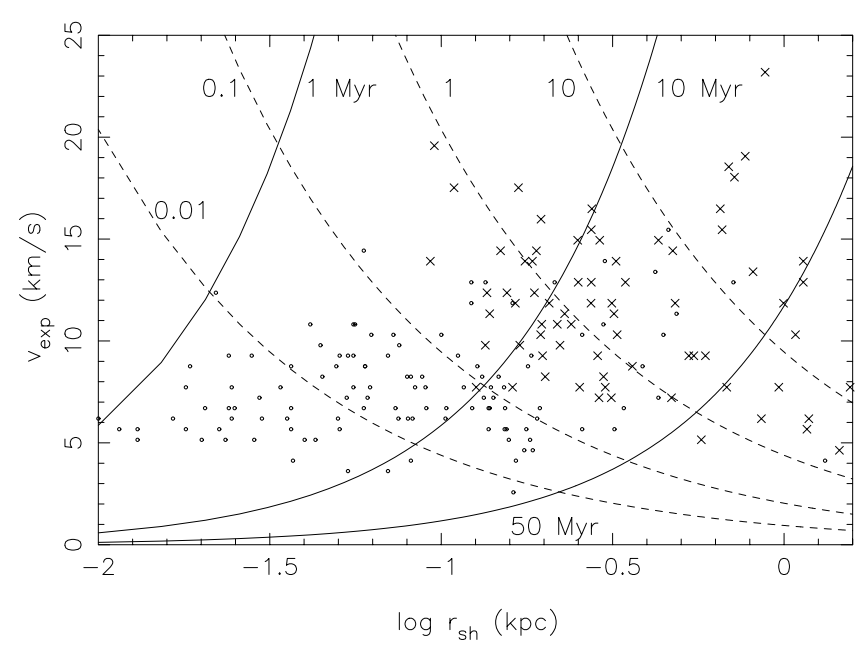

Fig. 8. Radius of shell vs. expansion velocity for shells in the 2nd quadrant. Lines are analytical solutions after Weaver et al. (1977). Solid lines are lines of constant expansion time $(1,10,50 \mathrm{Myr})$, dashed lines are lines of constant $L / n\left(0.01,0.1,1,10 \mathrm{SN} \mathrm{Myr}-1 / \mathrm{cm}^{-3}\right)$. Different symbols denote different energies of shells (according to Chevalier 1974), circles are shells with $E<E_{\mathrm{SN}}$, crosses are shells with $E \geq E_{\mathrm{SN}}$.

multiplied by a factor of $3 / 5$ to change them from the simple $r / v$ used in WB99 to the solution of Weaver et al. (1977). The differences are explained by different star formation histories and a higher rate of shell destruction by density waves in spiral arms.

A somewhat different behavior was found for the Magellanic Clouds. Kim et al. (1999) shows an $r_{\text {sh }}$ vs. $v_{\text {exp }}$ graph for the LMC. There is a population of young shells, but there are no shells larger than $600 \mathrm{pc}$. This is not caused by the missing short spacing data, as it has been shown by Kim et al. (2003) and Staveley-Smith et al. (2003): there were no new identifications of supershells when the Parkes and ATCA observations were combined. Shells larger than $600 \mathrm{pc}$ are intrinsically missing in the LMC. The mean age of shells in the LMC is 4.9 Myr.

This is similar to the mean age of shells in the SMC (Staveley-Smith et al. 1997), 5.4 Myr. The list of shells in the SMC also suffers from the lack of large shells, but this effect is not so severe: in the combined Parkes and ATCA data (Stanimirovic̀ et al. 1999) only three new supershells with $r_{\mathrm{sh}}>600 \mathrm{pc}$ were found. The behavior of the Magellanic Clouds is clearly different from that of the dwarfs IC 2574 and Ho II. The presence of young shells in the SMC is explained by significant star formation induced by interaction with the Milky Way, but the lack of old shells - all the SMC shells are younger than $30 \mathrm{Myr}$ - is somewhat puzzling since the last SF burst in the SMC happened 200 Myr ago (Stanimirovic̀ et al. 2004).

The mean age of shells in our list is $8.4 \mathrm{Myr}$. This value is influenced by different selection effects, i.e. the detection of old shells is much less complete than of young ones since old ones are less continuous and more fragmented. All shells with ages greater than $30 \mathrm{Myr}$ are found at galactocentric distances larger than $12 \mathrm{kpc}$ - one shell with an age of $30 \mathrm{Myr}$ is located at $R=12 \mathrm{kpc}$, the rest of the old shells are at distances greater

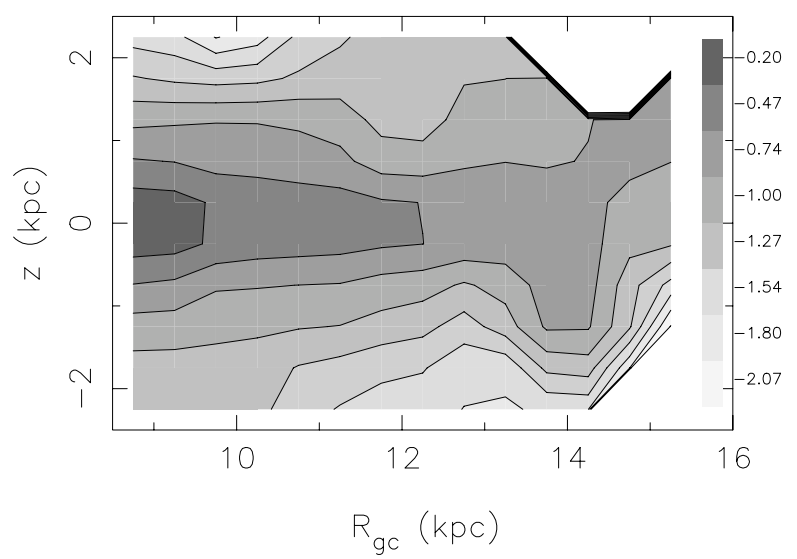

Fig. 9. An average H I density derived for shells at different positions in the Galaxy. The grey scale is logarithmic - the darkest color corresponds to $0.63 \mathrm{~cm}^{-3}$, lightest color to $0.01 \mathrm{~cm}^{-3}$, the white color denotes positions with no information about the density, i.e. without an $\mathrm{H}$ I shell.

than $16 \mathrm{kpc}$. The age distribution of shells is similar to that for spiral galaxies M 31 and M 33.

Our search algorithm is not very sensitive to fragmented structures, which may explain the lack of old shells. However, as we do not see any old shells at distances smaller than $12 \mathrm{kpc}$, while there are some at larger distances, we can at least say that the lifetime of shells is longer at greater galactocentric distances.

\subsection{H I density}

The original H I density at the place of a shell is calculated as the density corresponding to the mass missing in the H I hole. If that mass were evenly added to the hole, the structure would disappear and the observed H I column density would be equal to the average value around the shell. The maximum value found for an individual $\mathrm{HI}$ shell is $24 \mathrm{~cm}^{-3}$ for a very small nearby shell and $2.2 \mathrm{~cm}^{-3}$ for shells with a radius greater than $50 \mathrm{pc}$. The typical density is around $0.5 \mathrm{~cm}^{-3}$. We calculated an H I density $n$ in positions of all shells and made an average for each volume element of the galactic disc (Fig. 9). As expected, the density decreases with increasing $|z|$ and $R$ coordinates. These reflect the density gradients in the HI galaxy disk in combination with the shell size versus density effect: the observations show that shells expand more in a lower density medium. This is demonstrated in Fig. 10, where we plot sizes of shells $r_{\text {sh }}$ versus densities $n$.

\subsection{Energy}

The most widely used method to estimate the energy of H I shells is Chevalier's formula (Chevalier 1974),

$\frac{E_{\mathrm{tot}}}{\mathrm{erg}}=5.3 \times 10^{43}\left(\frac{n}{\mathrm{~cm}^{-3}}\right)^{1.12}\left(\frac{r_{\mathrm{sh}}}{\mathrm{pc}}\right)^{3.12}\left(\frac{v_{\mathrm{exp}}}{\mathrm{kms}^{-1}}\right)^{1.4}$

where $n$ is the density of the ambient medium, $r_{\mathrm{sh}}$ the radius of the shell and $v_{\exp }$ its expansion velocity. We take 


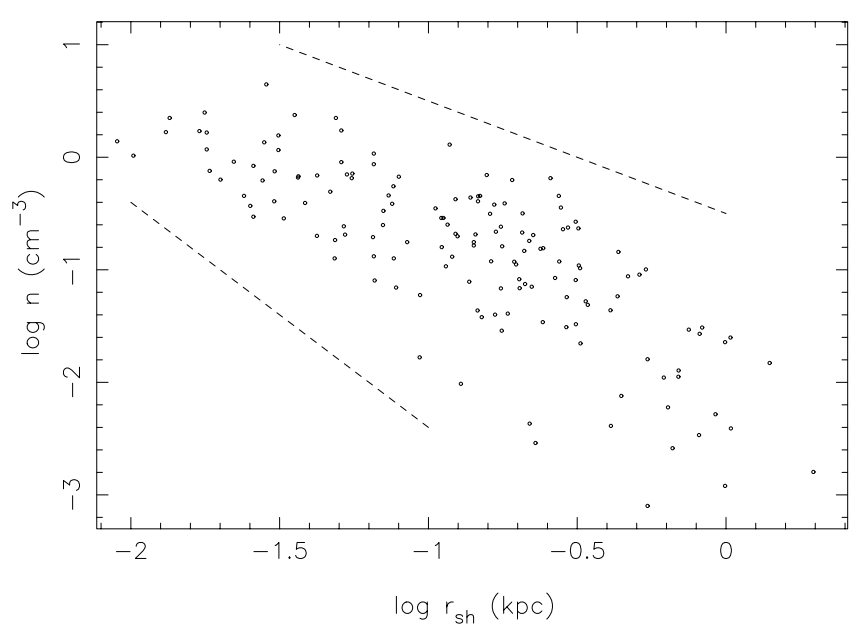

Fig. 10. The H I density versus size of the shells $r_{\mathrm{sh}}$. Two dashed auxiliary lines are power-laws with indices of -1 and -2 .

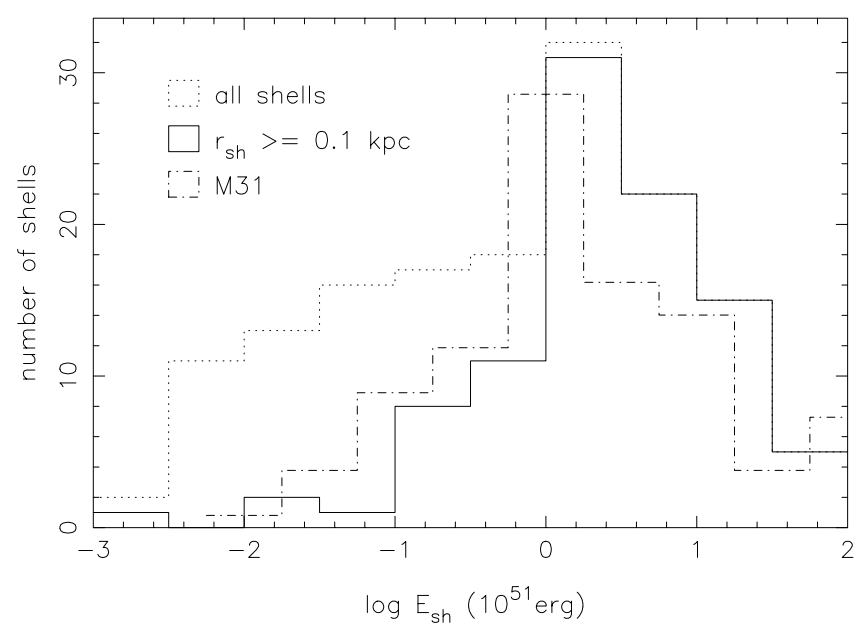

Fig. 11. The distribution of energy deposited in H I shells. The distribution of all shells (dotted line) and the distribution of shells with size $r_{\mathrm{sh}}>0.1 \mathrm{kpc}$ (solid line). The distribution for the M 31 galaxy (dashdotted line) is taken from Walter \& Brinks (1999); it is scaled to the Milky Way values.

$E_{\mathrm{SN}}=10^{51} \mathrm{erg}$. Energy estimations using Eq. (4) of smaller and less evolved shells are quite reliable, but for non-spherical shells it gives an underestimation of the energy that created the structure.

The distribution of the energies in $\mathrm{H}$ I shells according to the Eq. (4) is shown in Fig. 11. There are many low-energy structures $\left(E<0.1 E_{\mathrm{SN}}\right)$ that were probably not created by SN explosions. The majority of them are small with radii $r_{\mathrm{sh}}<$ 100 pc. Many of them are wind-blown shells. Even though Chevalier's Eq. (4) has the largest dependence on radius, many large shells do not belong to the most energetic structures since the density $n$ is systematically smaller for large compared to small shells; this influences the energy derived with Eq. (4) in the opposite direction to the size $r_{\mathrm{sh}}$.

There are only $12 \mathrm{H}$ I shells with an energy $E>20 E_{\mathrm{SN}}$ and one with an energy exceeding $100 E_{\mathrm{SN}}$ (its energy is $120 E_{\mathrm{SN}}$ ). This can be understood because we study the outer Milky Way and we do not observe many energetic sources there, at least not many OB associations. Bureau \& Carignan (2002) suggest that ram pressure due to a galaxy moving through the inter-galactic medium of a group or cluster can remove material from the edges of the holes and prevent it from refilling the holes' interiors. Thus, ram pressure enlarges preexisting holes and lowers the amount of energy necessary for their creation. Ram pressure and high velocity cloud impacts apply particularly at the galactic peripheries where the gas density and star formation rates are low.

We over-plot a scaled energy distribution of shells in M 31 (a dash-dotted line in Fig. 11) taken from WB99. They found that the distribution is very similar in the four galaxies (M 31, M 33, IC 2574, Ho II). The Milky Way distribution is similar as well: it has a maximum at the same value (around $1 E_{\mathrm{SN}}$ ) and the same decline for high energies. However, we detect more low-energy shells than were found in M 31, which may be partly a selection effect due to the larger distance to M 31, limiting the linear resolution. Another explanation is that the Milky Way creates more of them, or because our search algorithm is more sensitive than a visual inspection, upon which the M 31 distribution (and all others in WB99) is based. The mean energy of shells in M 31 is $15 E_{\mathrm{SN}}$, similar to M 33 (16 $\left.E_{\mathrm{SN}}\right)$, IC $2574\left(11 E_{\mathrm{SN}}\right)$ and Ho II (14 $\left.E_{\mathrm{SN}}\right)$. The mean energy of shells in the outer Milky Way is $3 E_{\mathrm{SN}}\left(4.7 E_{\mathrm{SN}}\right.$ for shells with $r_{\mathrm{sh}}>100 \mathrm{pc}$ ), this lower value is related to the extended lowenergy wing in the energy distribution function.

\section{Distribution of shells in the Milky Way from distance effect removed (DER) samples}

\subsection{Distance effect removed (DER) samples}

Due to our position inside the Milky Way our list of shells suffers from various distance-dependent selection effects. Intrinsically small structures are seen only in the vicinity of the Sun. When they are further out, they are too small to be either observed by the telescope or detected by our algorithm. On the other hand, large shells, when close to the Sun, are angularly extended and such objects are difficult to identify because they are too big for the field in which identifications take place, because of intervening material, and sometimes also because we usually see only walls of these structures and they are incomplete or fragmented. To avoid these problems we create "distance effect removed (DER) samples", where we include shells with dimensions and distances such that their angular dimensions fall into an interval $\left(\phi_{\min }, \phi_{\max }\right)=\left(1.5^{\circ} ; 15^{\circ}\right)$.

The lower limit $\phi_{\min }$ is the minimum dimension for identification, the upper limit $\phi_{\max }$ is smaller than the upper limit for identification $\left(45^{\circ}\right)$. This choice was made to ensure that we do not miss any structure in the selected interval. The range of heliocentric distances (or shell dimensions) is a free parameter. When processing a DER sample, we work with shells whose linear dimension $\Delta_{\mathrm{sh}}=2 r_{\mathrm{sh}}$ is $\Delta_{\mathrm{sh}} \in\left(\Delta_{\min }, \Delta_{\max }\right)$ and heliocentric distances $d$ are $d \in\left(d_{\min }, d_{\max }\right)$, where $\Delta_{\min }=$ $d_{\max } \tan \left(\phi_{\min }\right)$ and $\Delta_{\max }=d_{\min } \tan \left(\phi_{\max }\right)$. With this we ensure that the smallest shell detected at the largest distance $d_{\max }$ has an angular size equal to $\phi_{\min }$ and at the same time that the largest shell at the smallest distance $d_{\min }$ has an 

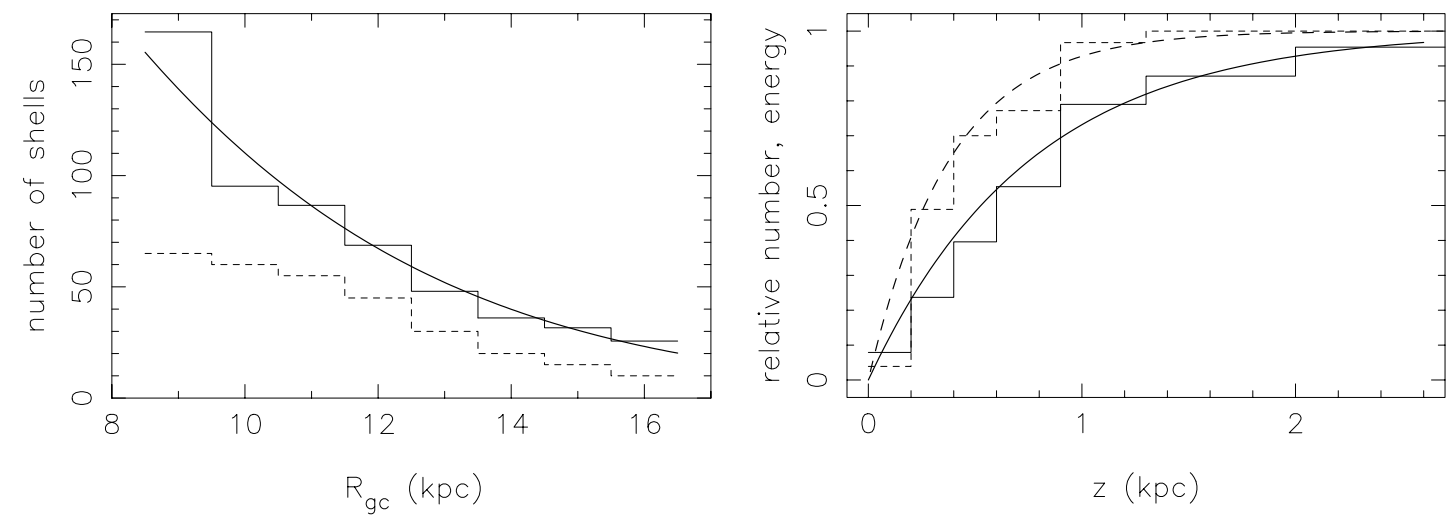

Fig. 12. The radial and $z$-distribution of $\mathrm{H}$ I shells. The left panel shows recomputed numbers of shells derived from a DER sample by multiplication with a geometric factor (see the text) at different galactocentric radii and an exponential fit with $\sigma_{\mathrm{gsh}}=3 \mathrm{kpc}$ (solid line). The dashed line gives the numbers from the DER sample (see the text). The right panel is the z-distribution of H I shells. Solid lines show the normalized cumulative number of shells at coordinates $z$ lower than a given value, dashed lines show the normalized energy in these shells. Binned data are observations, lines are exponential profiles with a scale length of $760 \mathrm{pc}$ (solid) and $380 \mathrm{pc}$ (dashed). Corresponding $H W H M \mathrm{~s}$ are 530 and $270 \mathrm{pc}$.

angular size equal to $\phi_{\max }$. We analyze different DER samples selected with different choices of minimal and maximal heliocentric distances $d_{\min }, d_{\text {max }}$. All shells in studied DER samples are larger than $100 \mathrm{pc}$, which avoids contamination by windblown bubbles and low-energy structures, and smaller than $700 \mathrm{pc}$ to avoid an incompletness in identifications of large evolved shells.

\subsection{Radial distribution}

It is generally thought that H I shells are mainly produced by SN explosions and other energetic activities in OB associations. Therefore their radial distribution should reflect the radial distribution of young stars and OB associations. Attempts to find a correlation between these distributions were made by Ehlerová \& Palouš (1996) for the Milky Way Galaxy and Palouš \& Ehlerová (1997) for M 31 and Holmberg II. The distribution of H I shells in the Milky Way was constructed from the list of Heiles (1979) and an exponential decrease of $\Sigma_{\text {gsh }} \propto \exp ^{-\left(\frac{R}{\sigma_{\text {gsh }}}\right)}$ was found, with $\Sigma_{\text {gsh }}$ being the surface density of $\mathrm{H}$ I shells, $R$ the galactocentric distance and $\sigma_{\mathrm{gsh}} \simeq 4.5 \mathrm{kpc}$ the radial scale. However, the effects of heliocentric distance were not taken into account properly. In this paper we will perform a similar analysis on our new and better-processed list of H I shells.

Another attempt to determine the galactic distribution of shells was made by McClure-Griffiths et al. (2002). Their list consists of shells from Heiles (1979) and their own identifications. They are mostly interested in the relation between shells and spiral arms.

We fit an exponential profile to the radial distribution of shells identified and described in this paper:

$\Sigma_{\mathrm{gsh}}(R)=\Sigma_{0 \mathrm{gsh}} \mathrm{e}^{-\frac{R}{\sigma_{\mathrm{gsh}}}}$,

where $R$ is the galactocentric distance and $\sigma_{\mathrm{gsh}}$ is the radial scale length. The number of shells $\mathrm{d} N$ at a given distance $R$ is

$\mathrm{d} N(R)=2 \pi R \Sigma_{\mathrm{gsh}}(R) \mathrm{d} R$.
The observed numbers of shells in intervals of galactocentric distances are multiplied by a geometric factor proportional to the ratio of the surface of the whole galactocentric ring and the surface of that part of the ring that lies in the studied heliocentric range. These recalculated numbers, which correspond to $\mathrm{d} N(R)$ in Eq. (6), are then fitted to the profile (5). The resulting radial scale length $\sigma_{\mathrm{gsh}}$ for the DER sample with $\Delta_{\min }=200 \mathrm{pc}$ and $\Delta_{\max }=800 \mathrm{pc}$ is

$\sigma_{\mathrm{gsh}}=3_{-1}^{+2} \mathrm{kpc}$.

We exclude shells with $r_{\mathrm{sh}}>400 \mathrm{pc}$ because DER samples including these large shells would have $d_{\min }>3 \mathrm{kpc}$. It would substantially reduce the number of shells in the interval of galactocentric distances $R_{\mathrm{GC}} \simeq(9,12) \mathrm{kpc}$ and increase the error of $\sigma_{\mathrm{gsh}}$. The fit, together with the observed and recalculated numbers of shells is shown in Fig. 12 (left panel).

The radial scale length of about $3 \mathrm{kpc}$ is comparable to that of the stellar disc. Luminosity models give values between 2.5-3.0 kpc (Kent et al. 1991, $\sigma=(3 \pm 0.5) \mathrm{kpc}$; Freudenreich $1998, \sigma=2.5 \mathrm{kpc})$. OB associations have a radial scale length of $1.8 \mathrm{kpc}$ (Bronfman et al. 2000). If the scale length of stars and $\mathrm{H}$ I shells differed substantially, it would mean that an important part of the shells is not connected to the stellar activity. This, however, is not the case. The derived radial scale length of $\mathrm{HI}$ shells roughly corresponds to the stellar scale length and is larger than the scale length of OB stars. The scale length of OB associations is based mainly on measurements in the inner Galaxy (for $R_{\mathrm{GC}} \in(0.5 ; 2.0) R_{\odot}$ with the majority of sources inside the solar circle), while the scale length of HI shells is derived only for the outer Galaxy. Therefore it is possible that the discrepancy is a result of a systematic difference between the inner and outer Galaxy. Another possible explanation is that a certain fraction of $\mathrm{HI}$ shells is created by a different mechanism than energy input from OB associations. 


\subsection{The distribution in the z-direction}

H I shells are concentrated towards the Galactic plane. One half of all shells lie in a $1-\mathrm{kpc}$ thin layer (the $H W H M$ is around $500 \mathrm{pc}$ ). The thickness of this layer increases slightly with galactocentric distance: the $H W H M$ for shells with $R \in$ $(9,11) \mathrm{kpc}$ is $530 \mathrm{pc}$ (see Fig. 12), for those with $R \in$ $(12,14) \mathrm{kpc}$ it is $590 \mathrm{pc}$. A more precise determination of this trend is not possible because of low number statistics at larger galactocentric distances.

Identified $\mathrm{H}$ I shells reside in a much thicker disc compared to the H I disc or even to the stellar disc. It may be partly due to our identification procedure, which shifts the shell centers artificially to lower density ends, i.e. to higher $z$-coordinates.

The average energy of shells (according to Eq. (4)) decreases with increasing coordinate $z$ (see Fig. 12). It is uncertain if this is a real physical effect (fewer energetic sources at high $z$ coordinates) or simply a consequence of the fact that the volume density decreases to higher values of $z$ and as a result the estimated energy is lower. The HWHM of the energy release is about half of that of the number density, i.e. around $250 \mathrm{pc}$, also increasing with increasing $R, 200$ pc for $R \in(9,11) \mathrm{kpc}$ and $230 \mathrm{pc}$ for $R \in(12,14) \mathrm{kpc}$.

The exponential or Gaussian fit of the $z$-distribution of $\mathrm{HI}$ shells is not as good as the radial one, but the exponential fit is better than Gaussian. The $H W H M$ derived from these fits is larger than observed. This means that there are more shells at high coordinates than would correspond to an exponential (Gaussian) profile, i.e. the distribution of shells has extended wings. Figure 12 (right panel) shows the $z$-distribution of shells in the DER sample with $\Delta_{\min }=200 \mathrm{pc}$ and $\Delta_{\max }=800 \mathrm{pc}$.

\subsection{Size distribution of shells}

Oey \& Clarke (1997; hereafter OC97) calculate the size distribution of shells from theoretical considerations of shell evolution. They found that the size distribution can be quite reliably approximated by a power law (2). They compare the size distributions of shells in the galaxies M 31, M 33, Holmberg II and SMC and find that the power-law representation generally fits the data well with $\alpha \in(2.1,2.7)$.

Figure 13 gives the size distribution together with three different power laws with $\alpha=1.1,2.1$ and 3.0. A fit of the DER sample with $\Delta_{\min }=200 \mathrm{pc}$ and $\Delta_{\max }=1400 \mathrm{pc}$ gives the average value of the index $\alpha$ around 2.1:

$\alpha=(2.1 \pm 0.4)$.

The spectrum seems to be flatter for smaller shells $(\alpha<2.1)$ and steeper for larger shells $(\alpha>2.1)$. There may be several regimes in the size distribution, one with $\alpha=1.1$, one with $\alpha=2.1$ and another with $\alpha=3.0$. The difference in the indices may be real or it may be an artifact of the identification method. Evolutionary effects should lead to an increase of $\alpha$ for large shells: some of the large old shells are destroyed, fragmented or otherwise distorted and therefore are more difficult to find. Merging and grouping of small shells produces a change in $\alpha$ between small and intermediate shells: instead of several small

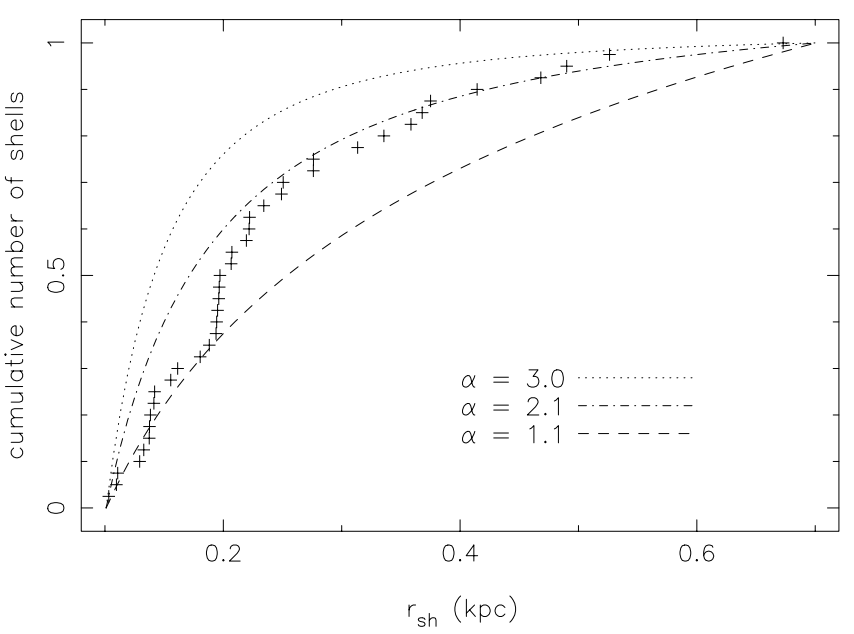

Fig. 13. The size distribution of HI shells. The number of shells with radius larger than $100 \mathrm{pc}$ and smaller than a given value of $r_{\mathrm{sh}}$ vs. the radius $r_{\mathrm{sh}}$ is plotted, plus a theoretical value for three different power-laws.

shells we see one larger shell. Our identification method (as discussed earlier) can underestimate the dimensions of extended shells in the $z(b)$ direction. Indeed, Fig. 5 shows that shells become more elongated in the $l$ directions with increasing $\Delta l$. This effect may also lead to higher $\alpha$ for large shells. An alternative explanation is that the completeness of our list is comparatively good for large shells, while it is worse for smaller shells, but this is unlikely as small shells are more regular and easier to find than large distorted structures.

The power-law index of the luminosity distribution of energy sources $\Phi(\mathcal{L})$ (e.g. OB associations) is given by OC97 as

$\Phi(\mathcal{L}) \propto \mathcal{L}^{-\beta}$,

where $\mathcal{L}$ is the luminosity of the source. OC97 give the relation between the power laws (2) and (9) as

$\alpha=2 \beta-1$,

which would imply from our size distribution fit

$\beta=(1.6 \pm 0.3)$.

However, the direct connection between sizes of shells and powering sources disregards the size-density relation shown in Fig. 10. It can be one of the systematic effects, which distinguishes between different types of galaxies. The indices $\alpha$ and $\beta$ are on the shallower end of the corresponding values given by OC97 for M 31, M 33, Holmberg II and SMC; $\alpha \in(2.1,2.7)$, $\beta \in(1.6,1.9)$. The index $\beta$ is flatter than 2 , which is the slope of the H II region luminosity function (Kennicutt et al. 1989) or OB association luminosity function (McKee \& Williams 1997). This lower value may be connected to the blow-out effect in the thin H I disc of the Milky Way. The sizes of shells powered by high $\mathcal{L}$ OB associations are smaller because a fraction of the energy leaks to high- $z$ distances. Blow-outs reduce the difference between the sizes of H I shells created by high and low $\mathcal{L}$ OB associations. The shell size distribution then leads to an artificially flatter luminosity function than that of OB associations. 


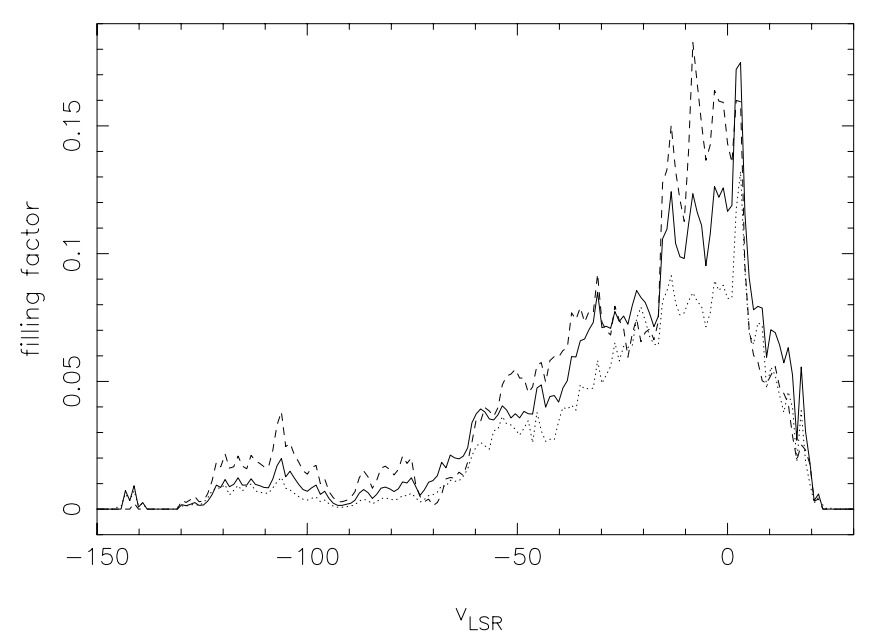

Fig. 14. The 2-D filling factor of $\mathrm{HI}$ holes in the $T_{\mathrm{B}}(l, b, v)$ datacube for $(l, b) \in\left(90^{\circ}, 180^{\circ} ;-10^{\circ},+10^{\circ}\right)$ (solid line), $(l, b) \in\left(90^{\circ}, 180^{\circ} ;-5^{\circ},+5^{\circ}\right)$ (dashed line), and $(l, b) \in$ $\left(90^{\circ}, 180^{\circ} ;-20^{\circ},+20^{\circ}\right)$ (dotted line) as a function of the radial velocity.

\section{Filling factor of $\mathrm{H}$ I shells}

The filling factor $f$ of HI shells is the total area $\left(f_{2 \mathrm{D}}\right)$ or volume $\left(f_{3 \mathrm{D}}\right)$ occupied by HI shells compared to the total area or volume of the galaxy. The derived filling factors are lower limits to intrinsic values, since some shells are missing (due to distance effects, incomplete identification, etc).

The 2-D filling factor as a function of radial velocity is shown in Fig. 14, where we show the results for strips along $b=0^{\circ}$. The profiles for the wider or narrower intervals of $b$ are similar. Neglecting distance effects, i.e. neglecting the fact that at different distances shells with different dimensions are not seen, the total filling factors in the 2nd galactic quadrant of the Milky Way are calculated. We get $f_{3 \mathrm{D}} \simeq 0.05$ and $f_{2 \mathrm{D}} \simeq 0.4$. We argue that neglecting distance effects we can approach to real, unbiased values. This is because filling factors are dominated by large shells, therefore the non-detection of small shells does not strongly influence the results.

\section{Summary}

We created an automatic method to search for $\mathrm{H}$ I shells in data cubes. The method is based on finding local minima in individual velocity channels. These $2 \mathrm{D}$ regions are then combined along the third dimension, the radial velocity axis, to create 3D objects. The spectrum through the center of the structure is analyzed. The method has the advantage of being independent of models of HI shells and therefore being widely applicable. Small shells (compared to the thickness of the disc) are more easily identified than large ones. Random brightness temperature fluctuations create structures with a small velocity extent, which are easily discarded when looking at spectra taken through the centers of the shells. We cannot detect shells that are formed by incomplete arcs: we detect only structures with a hole inside or completely encircled regions. We detect more shells than published by Heiles (1979) and other authors.
Some of the shells identified by us for the first time are as well defined as those previously catalogued.

In the whole Leiden-Dwingeloo survey we identify more than 600 structures. To avoid problems connected with a substantial overlap of shells in the inner Galaxy, we analyze only structures in the 2nd Galactic Quadrant. There are nearly 300 shells found there. From their list we create samples that compensate for the distance effect, i.e. the fact that we see small shells only in the vicinity of the Sun, and that angularly large shells are difficult to identify in the data.

Firstly, we compare shells in the Milky Way with shells in external galaxies. We find that galactic shells have similar properties to shells in M 31 and M 33. The majority of shells are $\sim 10$ Myr old and there are few shells older than $\sim 30 \mathrm{Myr}$. The energy distribution is similar to that of M 31, but we detect relatively more low-energy shells.

Secondly, we study the Galactic distribution of H I shells. The radial decrease in the surface density of shells in the outer parts of the galactic disk is exponential, with a scale length of about $3 \mathrm{kpc}$. This is comparable to the radial scale length of the stellar disc, but larger than the radial scale length of OB associations. The similarity between scale lengths of stars and HI shells supports the idea that most shells are connected to stars. The reason for the discrepancy between scale lengths of $\mathrm{HI}$ shells and OB associations is not clear, it may either reflect differences between the inner and outer Galaxy, or it may result from the fact that a fraction of shells in the outer Galaxy is created by a different mechanism than by an energy input from young and massive stars.

H I shells are concentrated towards the Galactic plane. Half of all shells lie in a 1-kpc thin layer (the HWHM is around $500 \mathrm{pc}$ ). The thickness of this layer increases slightly with galactocentric distance. The energy in shells is more concentrated toward the plane; half of all the energy is contained within the disc with a total thickness of $500 \mathrm{pc}$.

We then study the size distribution of HI shells. We find that a power-law function (2) with a slope $\alpha=2.1 \pm 0.4$ describes the observed distribution. $\alpha$ seems to be larger for larger shells and smaller (flatter) for smaller shells. We attribute the increase of $\alpha$ with dimension to evolutionary effects and the inability of the identification method to find distorted and fragmented structures. The size distribution with $\alpha=2.1$ is flatter, but comparable to the distributions found in the galaxies M 31, M 33, Holmberg II and SMC. The corresponding slope of the luminosity function of sources powering HI shells is $\beta=(1.6 \pm 0.3)$. This is also flatter than the observed distribution of $\mathrm{H}$ II regions or OB associations in the Milky Way. However, the size of shells also depends on the density of the ambient medium. Consequently, part of the effect may be due to a size-density relation observed in our shell sample.

The filling factors are derived for shells identified in the 2nd galactic quadrant. Due to incompleteness of the sample, the derived values $f_{3 \mathrm{D}}=0.05$ and $f_{2 \mathrm{D}}=0.4$ are lower limits to the intrinsic values.

With $r_{\mathrm{sh}}, v_{\mathrm{exp}}$, the surface density of the shell $\Sigma_{\mathrm{sh}}$ and with the speed of sound in the shell, it would be possible to estimate the gravitational stability of the shell and find out if it can trigger star formation. Some of the shells detected in the LMC, like 
the Sextant region near LMC IV (Efremov et al. 1999), probably triggered star formation. A discussion of shell triggering and a comparison between the Milky Way, LMC, Ho II and other galaxies will be published elsewhere.

We conclude that our automatic identification code is a powerful tool in studies of H I shells, which enabled us to perform the so far most extensive statistical study of shells in the Milky Way.

Acknowledgements. The authors gratefully acknowledge financial support by the Grant Agency of the Academy of Sciences of the Czech Republic under the grant No. B3003106 and support by the grant project of the Academy of Sciences of the Czech Republic No. K1048102. They would also like to express their thanks to Bruce G. Elmegreen for comments on how to improve the English and to the anonymous referee for suggestions on how to improve the paper.

\section{References}

Bronfman, L., Casassus, S., May, J., \& Nyman, L.-Å. 2000, A\&A, 358,521

Bureau, M., \& Carignan, C. 2002, AJ, 123, 1316

Chevalier, R. 1974, ApJ, 188, 501

Daigle, A., Joncas, G., Parizeau, M., \& Miville-Deschênes, M.-A. 2003, PASP, 115, 662

de Heij, V., Braun, R., \& Burton, W. B. 2002, A\&A, 391, 159

Hu, E. M. 1981, ApJ, 248, 119

Efremov, Yu. N., Ehlerová, S., \& Palouš, J. 1999, A\&A, 350, 457

Efremov, Yu. N., Elmegreen, B. G., \& Hodge, P. W. 1998, ApJ, 501, L163

Ehlerová, S., \& Palouš, J. 1996, A\&A, 313, 478

Ferrière, K. 1995, ApJ, 441, 281

Ferrière, K. 1998, ApJ, 503, 700

Freudenreich, H. T. 1998, ApJ, 492, 495

Hartmann, D., \& Burton, W. B. 1997, Atlas of Galactic Neutral Hydrogen (Cambridge University Press)
Heiles, C. 1979, ApJ, 229, 533

Heiles, C. 1984, ApJS, 55, 585

Heiles, C. 1990, ApJ, 354, 483

Heiles, C., \& Habing, H. J. 1974, A\&AS, 14, 1

Hu, E. 1981, ApJ, 248, 119

Kennicutt, R. C., Edgar, B. K., \& Hodge, P. W. 1989, ApJ, 337, 761

Kent, S. M., Dame, T. M., \& Fazio, G. 1991, ApJ, 378, 131

Kim, S., Dopita, M. A., Staveley-Smith, L., \& Bessell, M. S. 1999, AJ, 118, 2797

Loeb, A., \& Perma, R. 1998, ApJ, 503, L35

Mashchenko, S. Y., \& St-Louis, N. 2002, in ASP Conf. Proc., ed. A. F. J. Moffat, \& N. St-Louis, 260, 65

Mashchenko, S. Y., Thilker, D. A., \& Braun, R. 1999, A\&A, 343, 352

McClure-Griffiths, N. M., Dickey, J. M., Gaensler, B. M., \& Green, A. J. 2002, ApJ, 578, 176

McKee, C. F., \& Williams, J. P. 1997, ApJ, 476, 144

Oey, M. S., \& Clarke, C. J. 1997, MNRAS, 289, 570 (OC97)

Palouš, J., \& Ehlerová, S. 1997, in The Physics of Galactic Halos, ed. H. Lesch, R.-J. Dettmar, U. Mebold, \& R. Schlickeiser, 291

Slavin, J. D., \& Cox, D. P. 1993, ApJ, 417, 187

Stanimirovic̀, S., Staveley-Smith, L., Dickey, J. M., Sault, R. J., \& Snowden, S. L. 1999, MNRAS, 302, 417

Stanimirović, S., Staveley-Smith, L., \& Jones, P. A. 2004, ApJ, 604, 176

Staveley-Smith, L., Sault, R. J., Hatzidimitriou, D., Kesteven, M. J., \& McConnell, D. 1997, MNRAS, 289, 225

Staveley-Smith, L., Kim, S., Calabretta, M. R., Haynes, R. F., \& Kesteven, M. J. 2003, MNRAS, 339, 87

Tenorio-Tagle, G., \& Bodenheimer, P. 1988, ARA\&A, 26, 145

Thilker, D. A., Braun, R., \& Walterbos, R. A. M. 1998, A\&A, 332, 429

Walter, F., \& Brinks, E. 1999, AJ, 118, 273 (WB99)

Weaver, R., McCray, R., \& Castor, J. 1977, ApJ, 218, 377

Weaver, H., \& Williams, D. R. W. 1973, A\&AS, 8, 1

Wouterloot, J. G. A., Brand, J., Burton, W. B., \& Kwee, K. K. 1990, A\&A, 230, 21 


\section{Online Material}




\section{Appendix A: A comparison with other lists}

\section{A.1. Heiles}

Heiles' list of H I shells in the Milky Way (Heiles 1979) contains fourteen shells in the 2nd Galactic quadrant identified in the H I survey of Weaver \& Williams (1973). A visual inspection of the LDS shows that nine of the fourteen shells in the Heiles list are clearly and easily seen. Three shells are formed by partial walls and there are no well-defined holes. Two shells are completely open to the halo.

Seven of the nine Heiles structures were re-identified by our method. The remaining two were found in velocity channels but were later rejected because of unsuitable shapes of spectra. The derived properties of these nine structures (seven shells and two rejected candidates) are summarized in Table A.1 together with published values. The table shows a good correspondence both in angular dimensions and velocity extent. There are two cases where previous and newly found dimensions differ: GS091-04-69 and GS128+01-105. GS091-04-69 is a shell which has inner walls - it might consist of several smaller shells - and we do not identify all of them as parts of the structure. The other shell, GS128+01-105, is found to have a larger dimension in $l$ and a larger range in radial velocity than was previously thought (see Fig. A.1 - left column and also Table A.1). Judging by eye we think that in this case our derived dimensions are better.

Three shells with partial walls were not identified as shells with our automatic method. We doubt the existence of one of them, GS123+07-127, as there is only a scarce group of fragments at the indicated position. The second shell, GS103+05-137, is very fragmented and we do not have any identification at this position, but we find a shell at these $l, b$ coordinates in an adjacent velocity interval. According to Heiles, this shell is seen at velocities lower than $-123 \mathrm{kms}^{-1}$ and we see a structure in an interval of $(-123,-100) \mathrm{kms}^{-1}$. Very probably both identifications, Heiles' and ours, are part of the same structure. The third structure from the list, GS130+00+15 is a large $\left(35^{\circ}\right.$ in $l$ ) shell formed by a clearly observed wall, however this wall does not encircle the whole structure. The hole (the region of minimum in the temperature distribution) is not pronounced in this case.

The two open structures (GS117-07-67 and GS129-05-91) are formed by incomplete arcs and therefore cannot be found by our algorithm.

\section{A.2. $\mathrm{Hu}$}

Hu (1981) made a list of high latitude shells detected in the H I survey of Heiles \& Habing (1974). The high latitude shells in Hu's list are more difficult to see in LDS data; about half of them can barely be distinguished by eye or are simply not seen at all (this is connected to the method used for Hu's identifications). The $\mathrm{Hu}$ list contains twelve shells in the second quadrant. Our automatic procedure is able to recover $50 \%$ of them. Some shells from Hu's list are too small (No. 26a) or fragmented (No. 26). Others (No. 25 and 28) are not connected to any well-pronounced HI depressions.
Table A.1. A comparison of previously known shells (Heiles 1979) and our identifications. The first column gives the names of the structures, the second one gives dimensions as published by Heiles, the third column gives our dimensions $(\Delta l, \Delta b, \Delta v)$.

\begin{tabular}{lrr}
\hline \hline Name & $\begin{array}{r}\text { Heiles } \\
\mathrm{deg}^{2} \mathrm{~km} \mathrm{~s}^{-1}\end{array}$ & $\begin{array}{r}\text { this paper } \\
\mathrm{deg}^{2} \mathrm{~km} \mathrm{~s}^{-1}\end{array}$ \\
\hline GS090+02-115 & $4 \times 4 \times 16$ & $3 \times 4 \times 12$ \\
GS091-04-69 & $9 \times 10 \times 28$ & $3 \times 5 \times 33$ \\
GS091+02-101 & $4 \times 3 \times 12$ & $4 \times 4 \times 33$ \\
GS108-04-23 & $5 \times 11 \times 24$ & $7 \times 14 \times 20$ \\
GS128+01-105 & $7 \times 6 \times 4$ & $11 \times 5 \times 16$ \\
GS148-01+15 & $4 \times 4 \times 4$ & $3 \times 5 \times 10$ \\
GS152-04-41 & $4 \times 4 \times 12$ & $11 \times 9 \times 12$ \\
\hline GS095+04-113 & $10 \times 5 \times 20$ & $10 \times 6 \times 21$ \\
GS139-03-69 & $18 \times 10 \times 28$ & $13 \times 6 \times 22$ \\
\hline
\end{tabular}

\section{A.3. CGPS}

Our identification algorithm was tested on two fields of the CGPS, where two structures were found in the automatic search by Mashchenko \& St.-Louis (2002). In the two data subsets, which were smoothed to a lower angular resolution, we were able to recover structures Sh2-203 and Sh2-187 with almost identical positions, dimensions and expansion velocities.

\section{A.4. Summary}

We identified with an automatic algorithm searching in the 2nd galactic quadrant in LDS $50 \%$ of the structures identified as HI shells in a search in the Weaver \& Williams (1974) survey performed by eye by Heiles (1979), and 50\% of the high latitude shells discovered with a filtering method by $\mathrm{Hu}$ (1981) in the HI survey of Heiles \& Habing (1974). We did not find shells formed only by incomplete arcs, or not connected to a minimum in $l b$ maps. We found many more (nearly 300) shells than those contained in the Heiles and Hu's lists. Many of these newly identified shells are well defined (i.e. with a regular shape and good contrast against the background) and have properties (dimensions, expansion velocities) comparable to those previously found. An example of such a structure at the position: $\left(l_{\mathrm{c}}, b_{\mathrm{c}}, v_{\mathrm{LSR}}\right)=(145.4,2.1,-48.3)$ and $(\Delta l, \Delta b, \Delta v)=(6.0,4.5,10.3)$ is shown in Fig. A.1 (right column). Thus, it is surprising that they have not been previously discovered. 
S. Ehlerová and J. Palouš: H I shells in the outer Milky Way, Online Material p 3
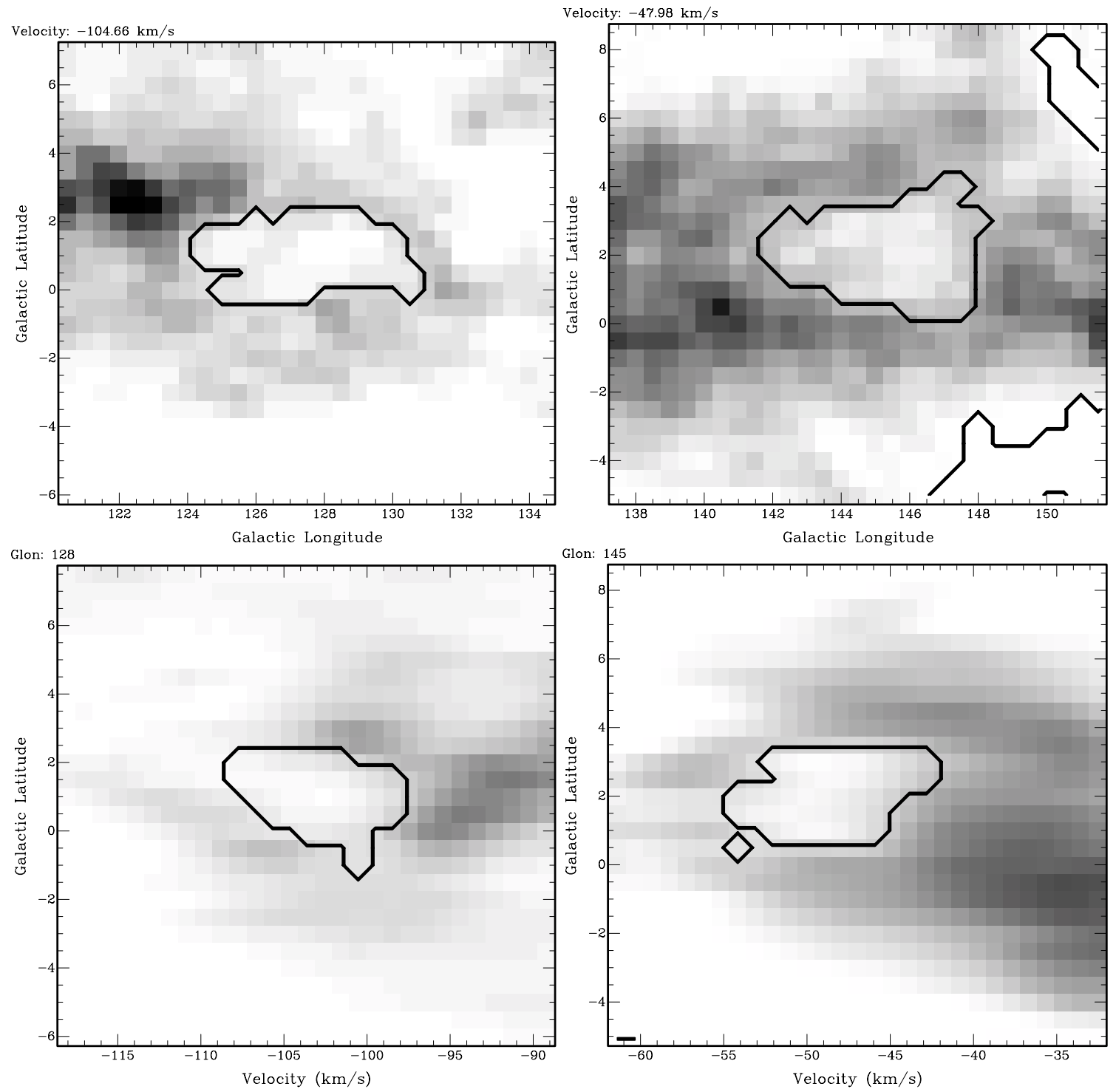

Fig. A.1. Re-identification of the shell GS 128+01-105 discovered by Heiles (1979) - left column, and a newly discovered shell GS145+02-048 (Table 1) - right column. The upper row shows $l b$-plots, the bottom row shows $b v$-diagrams. 